\title{
Subsequences of frames
}

\author{
by \\ R. VERShynin (Rehovot)
}

\begin{abstract}
Every frame in Hilbert space contains a subsequence equivalent to an orthogonal basis. If a frame is $n$-dimensional then this subsequence has length $(1-\varepsilon) n$. On the other hand, there is a frame which does not contain bases with brackets.
\end{abstract}

1. Introduction. The notion of frame goes back to R. Duffin and A. Schaeffer $[\mathrm{D}-\mathrm{S}]$ and has been studied extensively since then with relation to nonharmonic Fourier analysis (see [He]). From the geometrical point of view, a frame in a Hilbert space $H$ is the image of an orthonormal basis in a larger Hilbert space under an orthogonal projection onto $H$, up to equivalence $[\mathrm{Ho}]$ (the equivalence constant is called the frame constant). Since frames have nice representation properties (see [D-S], [A]), much attention has been paid to their subsequences that inherit these properties. The most interesting questions arise about subsequences equivalent to an orthogonal basis [Ho], [S], [C1], [C-C1]. P. Casazza [C2] proved that, given an $\varepsilon>0$, any $n$-dimensional frame whose norms are well bounded below contains a subsequence of length $(1-\varepsilon) n$ equivalent to an orthogonal basis (the constant of equivalence does not depend on $n$ ).

In the present paper this is proved for all frames, without restrictions on norms of the elements. If a frame is $n$-dimensional then it contains a subsequence of length $(1-\varepsilon) n$ which is $C$-equivalent to an orthogonal basis. Here $C$ depends only on the frame constant and $\varepsilon$. To put the result in other words, orthogonal projections in Hilbert space preserve orthogonal structure in almost the whole range. Namely, the image of an orthogonal basis under an orthogonal projection $P$ contains a subset of cardinality $(1-\varepsilon) \operatorname{rank}(P)$ which is $C(\varepsilon)$-equivalent to an orthogonal system. This is proved in Section 2.

2000 Mathematics Subject Classification: 46C05, 46B07.

This work was conducted at Friedrich-Schiller-Universität Jena and University of Missouri-Columbia. 
An infinite-dimensional version of this result is considered in Section 3. Every infinite-dimensional frame has an infinite subsequence equivalent to an orthogonal basis. However, for some frames this subsequence cannot be complete, as was shown by K. Seip $[\mathrm{S}]$ and P. Casazza and O. Christensen [C-C2]. This result is generalized in Section 4 by constructing a frame which does not contain bases with brackets. So our frame $\left(x_{j}\right)$ is "asymptotically indecomposable" in the following sense: if $\left(y_{j}\right)$ is any complete subsequence of $\left(x_{j}\right)$, then the distance from $\operatorname{span}\left(y_{j}\right)_{j \leq n}$ to $\operatorname{span}\left(y_{j}\right)_{j>n}$ tends to zero as $n \rightarrow \infty$.

In the rest of this section we recall standard definitions and simple known facts about frames. In what follows, $H$ will denote a separable Hilbert space, finite- or infinite-dimensional. Absolute constants will be denoted by $c_{1}, c_{2}, \ldots$ A sequence $\left(x_{j}\right)$ in $H$ is called a frame if there exist positive numbers $A$ and $B$ such that

$$
A\|x\|^{2} \leq \sum_{j}\left|\left\langle x, x_{j}\right\rangle\right|^{2} \leq B\|x\|^{2} \quad \text { for } x \in H .
$$

The number $(B / A)^{1 / 2}$ is called a constant of the frame. We call $\left(x_{j}\right)$ a tight frame if $A=B=1$.

Two sequences $\left(x_{j}\right)$ and $\left(y_{j}\right)$ in possibly different Banach spaces are called equivalent if there is an isomorphism $T:\left[x_{j}\right] \rightarrow\left[y_{j}\right]$ such that $T x_{j}$ $=y_{j}$ for all $j$. Here $\left[x_{j}\right]$ denotes the closed linear span of $\left(x_{j}\right)$. Let $c=$ $\|T\| \cdot\left\|T^{-1}\right\| ;$ then the sequences $\left(x_{j}\right)$ and $\left(y_{j}\right)$ are called $c$-equivalent.

The next observation (see $[\mathrm{Ho}]$ ) allows us to look at frames as at projections of the canonical vector basis $\left(e_{j}\right)$ in $l_{2}$.

Proposition 1. Let $\left(x_{n}\right)_{n=1}^{m}$ be a frame in $H$ with constant $c$, where $m$ can be infinity. Then there is an orthogonal projection $P$ in $l_{2}^{m}$ such that $\left(x_{n}\right)$ is c-equivalent to $\left(P e_{n}\right)$. Conversely, if $P$ is an orthogonal projection in $l_{2}^{m}$ onto a subspace $H$, then $\left(P e_{n}\right)_{n=1}^{m}$ is a tight frame in $H$.

Corollary 2. Let $\left(x_{n}\right)$ be a frame with constant $c$. Then $\left(x_{n}\right)$ is cequivalent to a tight frame.

Now we present another view on frames. We can regard them as columns of a row-orthogonal matrix (either finite or infinite).

Lemma 3. Let $n, m \in \mathbb{N} \cup \infty$ and $A$ be an $n \times m$ matrix whose rows are orthonormal. Then the columns of $A$ form a tight frame in $l_{2}^{n}$. Conversely, let $\left(x_{j}\right)_{j=1}^{m}$ be a frame in $H$. Then there exists an $n \times m$ matrix $A$ with $n=\operatorname{dim} H$ whose rows are orthonormal and such that the columns form a tight frame equivalent to $\left(x_{j}\right)$.

Proof. If $A$ is as above then $A^{*}$ acts as an isometric embedding of $l_{2}^{n}$ into $l_{2}^{m}$. Then $A$ acts as a quotient map in a Hilbert space, and we can regard it 
as an orthogonal projection. On the other hand, the columns of $A$ are equal to $A e_{j}$. Proposition 1 finishes the proof of the first statement. The converse can also be proved by this argument.

Lemma 4. Let $\left(x_{j}\right)$ be a tight frame in $H$. Then $\sum_{j}\left\|x_{j}\right\|^{2}=\operatorname{dim} H$ (which is possibly infinite).

Proof. By Proposition 1 we may assume that $H$ is a subspace of $l_{2}$ and $x_{j}=P e_{j}$, where $P$ is the orthogonal projection in $l_{2}$ onto $H$. Then the Hilbert-Schmidt norm $\|P\|_{\mathrm{HS}}$ is $\left(\sum_{j}\left\|x_{j}\right\|^{2}\right)^{1 / 2}$. On the other hand, $\|P\|_{\mathrm{HS}}=$ $(\operatorname{dim} H)^{1 / 2}$.

2. Finite-dimensional frames. In this section we prove

Theorem 5. There is a function $h: \mathbb{R}_{+} \rightarrow \mathbb{R}_{+}$such that the following holds. Suppose $\left(x_{j}\right)$ is an n-dimensional frame with constant $c$. Then for every $\varepsilon>0$ there is a set $\sigma$ of indices with $|\sigma|>(1-\varepsilon) n$ such that the system $\left(x_{j}\right)_{j \in \sigma}$ is $C$-equivalent to an orthogonal basis, where $C=h(\varepsilon) c$.

We will need a result of A. Lunin on norms of restrictions of operators to coordinate subspaces $([\mathrm{L}]$; for improvements see $[\mathrm{K}-\mathrm{Tz}])$.

Theorem 6 (A. Lunin). Let $T: l_{2}^{m} \rightarrow l_{2}^{n}$ be a linear operator. Then there is a set $\sigma \subset\{1, \ldots, m\}$ with $|\sigma|=n$ such that

$$
\left\|\left.T\right|_{\mathbb{R}^{\sigma}}\right\| \leq c_{1} \sqrt{n / m}\|T\| .
$$

Given an $h>0$, a system of vectors $\left(x_{j}\right)$ in a Hilbert space is called $h$-Hilbertian if

$$
\left\|\sum_{j} a_{j} x_{j}\right\| \leq h\left(\sum_{j}\left|a_{j}\right|^{2}\right)^{1 / 2}
$$

for all sequences $\left(a_{j}\right)$ of scalars. Then Theorem 6 can be reformulated as follows. Suppose $\left(x_{j}\right)_{1 \leq j \leq m}$ is a 1-Hilbertian system in $l_{2}^{n}$. Then there is a set $\sigma \subset\{1, \ldots, m\}$ with $|\sigma|=n$ such that $\left(\sqrt{m / n} x_{j}\right)_{j \in \sigma}$ is $c_{1}$-Hilbertian.

Next, we will use a result of J. Bourgain and L. Tzafriri on invertibility of large submatrices ([B-Tz], Theorem 1.2):

Theorem 7 (J. Bourgain, L. Tzafriri). Let $T: l_{2}^{n} \rightarrow l_{2}^{n}$ be a linear operator such that $\left\|T e_{j}\right\|=1$ for all $j$. Then there is a set $\sigma \subset\{1, \ldots, n\}$ with $|\sigma| \geq c_{2} n /\|T\|^{2}$ such that

$$
\|T x\| \geq c_{2}\|x\| \quad \text { for every } x \in \mathbb{R}^{\sigma} .
$$

Given a $b>0$, a system of vectors $\left(x_{j}\right)$ in a Hilbert space is called $b$-Besselian if

$$
b\left\|\sum_{j} a_{j} x_{j}\right\| \geq\left(\sum_{j}\left|a_{j}\right|^{2}\right)^{1 / 2}
$$


for all sequences $\left(a_{j}\right)$ of scalars. Then Theorem 7 can be reformulated as follows. Suppose $\left(x_{j}\right)_{1 \leq j \leq n}$ is an $h$-Hilbertian system in $l_{2}^{n}$ and $\left\|x_{j}\right\| \geq \alpha$ for all $1 \leq j \leq n$. Then there is a set $\sigma \subset\{1, \ldots, n\}$ with $|\sigma| \geq c_{2}(\alpha / h)^{2} n$ such that the system $\left(\alpha^{-1} x_{j}\right)_{j \in \sigma}$ is $c_{3}$-Besselian.

Clearly, every tight frame is 1-Hilbertian.

LEMma 8. Let $\left(y_{j}\right)_{1 \leq j \leq m}$ be a tight frame in $l_{2}^{n}$ with $\left\|y_{j}\right\|=\sqrt{n / m}$ for all $j$. Let $P$ be a $k$-dimensional orthogonal projection in $l_{2}^{n}$. Then for $\delta>0$,

$$
\left|\left\{j:\left\|(I-P) y_{j}\right\| \geq \delta \sqrt{n / m}\right\}\right| \geq\left(1-\delta^{2}-k / n\right) m .
$$

Proof. Let $\tau=\left\{j:\left\|(I-P) y_{j}\right\| \geq \delta \sqrt{n / m}\right\}$. Since $\left((I-P) y_{j}\right)_{1 \leq j \leq m}$ is a tight frame in the $(n-k)$-dimensional space $(I-P) l_{2}^{n}$, Lemma 4 yields

$$
\begin{aligned}
n-k & =\sum_{j=1}^{m}\left\|(I-P) y_{j}\right\|^{2} \leq \sum_{j \in \tau}\left\|y_{j}\right\|^{2}+\sum_{j \in \tau^{\mathrm{c}}}\left\|(I-P) y_{j}\right\|^{2} \\
& \leq|\tau| \cdot(n / m)+m \cdot \delta^{2}(n / m)=\left(|\tau| / m+\delta^{2}\right) n .
\end{aligned}
$$

The required estimate follows.

Now we proceed to the proof of Theorem 5. As in P. Casazza's proof [C2], the set $\sigma$ will be constructed by an iteration procedure. Our proof consists of several parts.

I. Splitting. By Corollary 2, we may assume that the frame $\left(x_{j}\right) \subset l_{2}^{n}$ is tight and all of its terms are nonzero. First, we split $\left(x_{j}\right)$ to get almost equal norms of the terms. Note that if we substitute any member $x_{j}$ of the frame by $k$ elements $x_{j} / \sqrt{k}, \ldots, x_{j} / \sqrt{k}$, we still get a tight frame. Fix $\nu>0$. Splitting each $x_{j}$ as above, we obtain a new tight frame $\left(y_{j}\right)_{1 \leq j \leq m}$ such that

(i) elements of $\left(y_{j}\right)$ are multiples of the ones from $\left(x_{j}\right)$;

(ii) there is a $\lambda>0$ such that $\lambda \leq\left\|y_{j}\right\| \leq(1+\nu) \lambda$ for all $j=1, \ldots, m$.

The constant $\lambda$ can be evaluated using Lemma 4 :

$$
(1+\nu)^{-1} \sqrt{n / m} \leq\left\|y_{j}\right\| \leq(1+\nu) \sqrt{n / m} \text { for } j=1, \ldots, m .
$$

Clearly, it is enough to prove the theorem for $\left(y_{j}\right)$ instead of $\left(x_{j}\right)$. We can choose the parameter $\nu=\nu(\varepsilon)>0$ arbitrarily small. To make the proof more readable, we simply assume that $\nu=0$. The reader will easily adjust the argument to the general case. So we have

$$
\left\|y_{j}\right\|=\sqrt{n / m}, \quad j=1, \ldots, m .
$$

We can also assume that $(\varepsilon / 2) m \geq n$. 
II. Iterative construction. Let $\delta=\sqrt{\varepsilon / 2}$.

SteP 1. Set $\tau_{0}=\{1, \ldots, m\}$. The system $\left(y_{j}\right)_{j \in \tau_{0}}$ is 1-Hilbertian. Lunin's theorem yields the existence of a set $\sigma_{1}^{\prime} \subset \tau_{0}$ with $\left|\sigma_{1}^{\prime}\right|=n$ such that the system $\left(\sqrt{m / n} y_{j}\right)_{j \in \sigma_{1}^{\prime}}$ is $c_{1}$-Hilbertian.

Note that $\left\|\sqrt{m / n} y_{j}\right\|=1$ for $j \in \sigma_{1}^{\prime}$. Then Bourgain-Tzafriri's theorem gives us a set $\sigma_{1} \subset \sigma_{1}^{\prime}$ with $\left|\sigma_{1}\right| \geq\left(c_{2} / c_{1}^{2}\right) n$ such that the system $\left(\sqrt{m / n} y_{j}\right)_{j \in \sigma_{1}}$ is $c_{3}$-Besselian.

So we have already found a subsequence $\left(y_{j}\right)_{j \in \sigma_{1}}$ of length proportional to $n$ which is well equivalent to an orthogonal basis. If $\left|\sigma_{1}\right| \geq(1-\varepsilon) n$, then we are done. Otherwise we proceed to the next step.

SteP 2. Let $P_{1}$ be the orthogonal projection in $l_{2}^{n}$ onto $\left[y_{j}\right]_{j \in \sigma_{1}}$. Let

$$
\tau_{1}=\left\{j:\left\|\left(I-P_{1}\right) y_{j}\right\| \geq \delta \sqrt{n / m}\right\} .
$$

Clearly, $\tau_{1} \subset \sigma_{1}^{\mathrm{c}}$. By Lemma 8 ,

$$
\left|\tau_{1}\right| \geq\left(1-\delta^{2}-\left|\sigma_{1}\right| / n\right) m
$$

As $\left|\sigma_{1}\right|<(1-\varepsilon) n$, we obtain

$$
\left|\tau_{1}\right|>\left(1-\delta^{2}-(1-\varepsilon)\right) m=(\varepsilon / 2) m .
$$

The system $\left(y_{j}\right)_{j \in \tau_{1}}$ is 1-Hilbertian and $\left|\tau_{1}\right| \geq n$ by the choice of $m$. Lunin's theorem yields the existence of a set $\sigma_{2}^{\prime} \subset \tau_{1}$ with $\left|\sigma_{2}^{\prime}\right|=n$ such that

the system $\left(\sqrt{\left|\tau_{1}\right| / n} y_{j}\right)_{j \in \sigma_{2}^{\prime}}$ is $c_{1}$-Hilbertian.

Then the system $\left(\sqrt{\left|\tau_{1}\right| / n}\left(I-P_{1}\right) y_{j}\right)_{j \in \sigma_{2}^{\prime}}$ is also $c_{1}$-Hilbertian. By the definition of $\tau_{1}$, it has not too small norms:

$$
\left\|\sqrt{\left|\tau_{1}\right| / n}\left(I-P_{1}\right) y_{j}\right\| \geq \delta \sqrt{\left|\tau_{1}\right| / m}, \quad j \in \sigma_{2}^{\prime} .
$$

Then Bourgain-Tzafriri's theorem gives us a set $\sigma_{2} \subset \sigma_{2}^{\prime}$ with

$$
\left|\sigma_{2}\right| \geq c_{2}\left(\delta^{2}\left|\tau_{1}\right| /\left(m c_{1}^{2}\right)\right) n \geq\left(c_{2} / c_{1}^{2}\right) \delta^{2}\left(\left(1-\delta^{2}\right) n-\left|\sigma_{1}\right|\right)
$$

such that

the system $\left(\sqrt{m / n}\left(I-P_{1}\right) y_{j}\right)_{j \in \sigma_{2}}$ is $\left(c_{3} \delta^{-1}\right)$-Besselian.

If $\left|\sigma_{1}\right|+\left|\sigma_{2}\right| \geq(1-\varepsilon) n$, then we stop. Otherwise we proceed to the next step.

STEP $k+1$. We assume that the sets $\sigma_{1}, \ldots, \sigma_{k}$ are already constructed and

$$
\sum_{i=1}^{k}\left|\sigma_{i}\right|<(1-\varepsilon) n .
$$

Let $P_{k}$ be the orthogonal projection in $l_{2}^{n}$ onto $\left[y_{j}\right]_{j \in \sigma_{1} \cup \ldots \cup \sigma_{k}}$. Let 


$$
\tau_{k}=\left\{j:\left\|\left(I-P_{k}\right) y_{j}\right\| \geq \delta \sqrt{n / m}\right\} .
$$

Clearly, $\tau_{k} \subset\left(\sigma_{1} \cup \ldots \cup \sigma_{k}\right)^{\mathrm{c}}$. By Lemma 8,

$$
\left|\tau_{k}\right| \geq\left(1-\delta^{2}-\sum_{i=1}^{k}\left|\sigma_{i}\right| / n\right) m
$$

By (1),

$$
\left|\tau_{k}\right|>\left(1-\delta^{2}-(1-\varepsilon)\right) m=(\varepsilon / 2) m
$$

The system $\left(y_{j}\right)_{j \in \tau_{k}}$ is 1-Hilbertian and $\left|\tau_{k}\right| \geq n$ by the choice of $m$. Lunin's theorem yields the existence of a set $\sigma_{k+1}^{\prime} \subset \tau_{k}$ with $\left|\sigma_{k+1}^{\prime}\right|=n$ such that

the system $\left(\sqrt{\left|\tau_{k}\right| / n} y_{j}\right)_{j \in \sigma_{k+1}^{\prime}}$ is $c_{1}$-Hilbertian.

Then the system $\left(\sqrt{\left|\tau_{k}\right| / n}\left(I-P_{k}\right) y_{j}\right)_{j \in \sigma_{k+1}^{\prime}}$ is also $c_{1}$-Hilbertian. By the definition of $\tau_{k}$, it has not too small norms:

$$
\left\|\sqrt{\left|\tau_{k}\right| / n}\left(I-P_{k}\right) y_{j}\right\| \geq \delta \sqrt{\left|\tau_{k}\right| / m}, \quad j \in \sigma_{k+1}^{\prime} .
$$

Then Bourgain-Tzafriri's theorem gives us a set $\sigma_{k+1} \subset \sigma_{k+1}^{\prime}$ with

$$
\left|\sigma_{k+1}\right| \geq c_{2}\left(\delta^{2}\left|\tau_{k}\right| /\left(m c_{1}^{2}\right)\right) n \geq\left(c_{2} / c_{1}^{2}\right) \delta^{2}\left(\left(1-\delta^{2}\right) n-\sum_{i=1}^{k}\left|\sigma_{i}\right|\right)
$$

such that

$$
\text { the system }\left(\sqrt{m / n}\left(I-P_{k}\right) y_{j}\right)_{j \in \sigma_{k+1}} \text { is }\left(c_{3} \delta^{-1}\right) \text {-Besselian. }
$$

If $\sum_{i=1}^{k+1}\left|\sigma_{i}\right| \geq(1-\varepsilon) n$, then we stop. Otherwise we proceed to the next step.

III. When we stop. Let $k_{0}$ be the number of the last step, that is, the smallest integer such that

$$
\sum_{i=1}^{k_{0}}\left|\sigma_{i}\right| \geq(1-\varepsilon) n
$$

We claim that $k_{0}$ exists and there is a function $K(\varepsilon)$ such that $k_{0} \leq K(\varepsilon)$. Indeed, let $K(\varepsilon)=\left[4 c_{1}^{2} c_{2}^{-1} \varepsilon^{-2}\right]+2$. If the claim were not true, then

$$
\sum_{i=1}^{k}\left|\sigma_{i}\right|<(1-\varepsilon) n \quad \text { for } k=1, \ldots, K(\varepsilon) .
$$

Then by (2) for all $k=2, \ldots, K(\varepsilon)$,

$$
\left|\sigma_{k}\right| \geq\left(c_{2} / c_{1}^{2}\right) \delta^{2}\left(\left(1-\delta^{2}\right)-(1-\varepsilon)\right) n=\left(c_{2} / c_{1}^{2}\right)\left(\varepsilon^{2} / 4\right) n
$$


Thus

$$
\sum_{i=1}^{K(\varepsilon)}\left|\sigma_{i}\right| \geq(K(\varepsilon)-1) \cdot\left(c_{2} / c_{1}^{2}\right)\left(\varepsilon^{2} / 4\right) n \geq n .
$$

This contradiction proves the claim.

Now set $\sigma=\sigma_{1} \cup \ldots \cup \sigma_{k_{0}}$; then $|\sigma|>(1-\varepsilon) n$. To complete the proof of the theorem, it remains to check that the system $\left(\sqrt{m / n} y_{j}\right)_{j \in \sigma}$ is well equivalent to an orthonormal basis.

IV. Equivalence to the orthogonal basis within blocks $\sigma_{k}$. Recall that for every $k<k_{0}$ the size of $\tau_{k}$ is comparable to $m$, namely $\left|\tau_{k}\right| \geq(\varepsilon / 2) m$. Then we conclude from the construction the existence of functions $c_{1}(\varepsilon)$ and $c_{2}(\varepsilon)$ such that for every $k=1, \ldots, k_{0}$,

$$
\text { the system }\left(\sqrt{m / n} y_{j}\right)_{j \in \sigma_{k}} \text { is } c_{1}(\varepsilon) \text {-Hilbertian, }
$$

$$
\text { the system }\left(\sqrt{m / n}\left(I-P_{k-1}\right) y_{j}\right)_{j \in \sigma_{k}} \text { is } c_{2}(\varepsilon) \text {-Besselian. }
$$

V. The system $\left(\sqrt{m / n} y_{j}\right)_{j \in \sigma}$ is h-Hilbertian for some function $h=$ $h(\varepsilon)$. Indeed, fix scalars $\left(a_{j}\right)_{j \in \sigma}$ such that $\sum_{j \in \sigma}\left|a_{j}\right|^{2}=1$. Then

$$
\begin{aligned}
\left\|\sum_{j \in \sigma} a_{j}\left(\sqrt{m / n} y_{j}\right)\right\| & \leq \sum_{k=1}^{k_{0}}\left\|\sum_{j \in \sigma_{k}} a_{j}\left(\sqrt{m / n} y_{j}\right)\right\| \\
& \leq \sqrt{k_{0}}\left(\sum_{k=1}^{k_{0}}\left\|\sum_{j \in \sigma_{k}} a_{j}\left(\sqrt{m / n} y_{j}\right)\right\|^{2}\right)^{1 / 2} \\
& \leq \sqrt{k_{0}} c_{1}(\varepsilon)\left(\sum_{k=1}^{k_{0}} \sum_{j \in \sigma_{k}}\left|a_{j}\right|^{2}\right)^{1 / 2} \quad \text { by }(3) \\
& =\sqrt{K(\varepsilon)} c_{1}(\varepsilon) .
\end{aligned}
$$

VI. The system $\left(\sqrt{m / n} y_{j}\right)_{j \in \sigma}$ is $b$-Besselian for some function $b=$ $b(\varepsilon)$. We follow P. Casazza [C2]. Choose $r=r(\varepsilon)>2$ large enough (to be specified later). Let $a=a(\varepsilon)>0$ be such that $r^{k_{0}+1} a<1$. Fix scalars $\left(a_{j}\right)_{j \in \sigma}$ such that $\sum_{j \in \sigma}\left|a_{j}\right|^{2}=1$. Suppose

(5) $1 \leq k^{\prime} \leq k_{0}$ is the largest integer such that

$$
\left(\sum_{j \in \sigma_{k^{\prime}}}\left|a_{j}\right|^{2}\right)^{1 / 2} \geq r^{k_{0}-k^{\prime}} a \text {. }
$$

The $k^{\prime}$ must exist, since otherwise

$$
\left(\sum_{j \in \sigma}\left|a_{j}\right|^{2}\right)^{1 / 2} \leq \sum_{k=1}^{k_{0}}\left(\sum_{j \in \sigma_{k}}\left|a_{j}\right|^{2}\right)^{1 / 2} \leq \sum_{k=1}^{k_{0}} r^{k} a \leq r^{k_{0}+1} a<1,
$$

contradicting the choice of $a$. We have 


$$
\begin{aligned}
\| \sum_{j \in \sigma} a_{j}( & \left.\sqrt{m / n} y_{j}\right) \| \\
\geq & \left\|\sum_{k=1}^{k^{\prime}} \sum_{j \in \sigma_{k}} a_{j}\left(\sqrt{m / n} y_{j}\right)\right\|-\sum_{k=k^{\prime}+1}^{k_{0}}\left\|\sum_{j \in \sigma_{k}} a_{j}\left(\sqrt{m / n} y_{j}\right)\right\| \\
\geq & \left\|\left(I-P_{k^{\prime}-1}\right) \sum_{k=1}^{k^{\prime}} \sum_{j \in \sigma_{k}} a_{j}\left(\sqrt{m / n} y_{j}\right)\right\| \\
\geq & \left\|\sum_{j \in \sigma_{k^{\prime}}} a_{j}\left(\sqrt{m / n}\left(I-P_{k^{\prime}-1}\right) y_{j}\right)\right\|-c_{1}(\varepsilon) \sum_{k=k^{\prime}+1}^{k_{0}} r^{k_{0}-k} a \\
\geq & c_{2}(\varepsilon)^{-1}\left(\sum_{j \in \sigma_{k^{\prime}}}\left|a_{j}\right|^{2}\right)^{1 / 2}-c_{1}(\varepsilon) \frac{r^{k_{0}-k^{\prime}}}{r-1} a^{1 / 2} \text { by }(4) \\
\geq & \left(c_{2}(\varepsilon)^{-1}-c_{1}(\varepsilon)(r-1)^{-1}\right) r^{k_{0}-k^{\prime}} a \quad \text { by }(5) \\
\geq & \left(c_{2}(\varepsilon)^{-1}-c_{1}(\varepsilon)(r-1)^{-1}\right) a . \quad \text { by }
\end{aligned}
$$

If $r$ was chosen so that $c_{2}(\varepsilon)^{-1}-c_{1}(\varepsilon)(r-1)^{-1}>c_{2}(\varepsilon)^{-1} / 2$, we are done. The proof is complete.

REMARK 1 . $C$ tends to 1 as $\varepsilon \rightarrow 1$. This is a consequence of a restriction theorem $[\mathrm{K}-\mathrm{Tz}]$ which we use in the following special case (see also [B-Tz], Theorem 1.6).

Theorem 9 (B. Kashin, L. Tzafriri). Let $T$ be a linear operator in $l_{2}^{n}$ with 0 's on the diagonal and $\|T\|=1$. Let $1 / n \leq \delta<1$. Then there exists $a$ set $\sigma \subset\{1, \ldots, n\}$ with $|\sigma| \geq \delta n / 4$ for which

$$
\left\|R_{\sigma} T R_{\sigma}\right\| \leq c_{5} \delta^{1 / 2}
$$

First, Theorem 5 gives us a set $\sigma_{1}$ of indices with $\left|\sigma_{1}\right| \geq n / 2$ such that the system $\left(x_{j} /\left\|x_{j}\right\|\right)_{j \in \sigma_{1}}$ is $c_{6} c$-equivalent to the canonical vector basis of $l_{2}^{\sigma_{1}}$. Let $\delta=1-\varepsilon$ and $z_{j}=x_{j} /\left\|x_{j}\right\|$ for $j \in \sigma_{1}$. Consider the linear operator $T$ in $l_{2}^{\sigma_{1}}$ which sends $e_{j}$ to $z_{j}$ for $j \in \sigma_{1}$. Then the operator $T^{*} T-I$ has 0 's on the diagonal and is of norm at most $2 c_{6}^{2} c^{2}$. Applying Theorem 9 we get a set $\sigma \subset \sigma_{1}$ with $\sigma \geq \delta\left|\sigma_{1}\right| / 4$ such that for any sequence $\left(a_{j}\right)$ of scalars with $\sum_{j \in \sigma}\left|a_{j}\right|^{2}=1$,

$$
\left\|\left\langle\left(T^{*} T-I\right) \sum_{j \in \sigma} a_{j} e_{j}, \sum_{j \in \sigma} a_{j} e_{j}\right\rangle\right\| \leq\left(2 c_{6}^{2} c^{2}\right) c_{5} \delta^{1 / 2}=c_{7} c^{2} \delta^{1 / 2} .
$$


Thus

$$
\left.\left|\left\langle\sum_{j \in \sigma} a_{j} z_{j}, \sum_{j \in \sigma} a_{j} z_{j}\right\rangle-\sum_{j \in \sigma}\right| a_{j}\right|^{2} \mid \leq c_{7} c^{2} \delta^{1 / 2} .
$$

Therefore the sequence $\left(z_{j}\right)_{j \in \sigma}$ is $g(\delta)$-equivalent to $\left(e_{j}\right)_{j \in \sigma}$ for a function $g(\delta)$ which tends to 1 as $\delta \rightarrow 0$. This proves Remark 1 .

REMark 2. $h(\varepsilon)$ tends to infinity as $\varepsilon \rightarrow 0$. This is verified for the following tight frame $\left(x_{j}\right)_{1 \leq j \leq n+1}, n \geq 2$, considered by P. Casazza and O. Christensen in [C-C2]:

$$
\begin{aligned}
x_{j} & =e_{j}-n^{-1} \sum_{j=1}^{n} e_{j} \quad \text { for } j=1, \ldots, n ; \\
x_{n+1} & =n^{-1 / 2} \sum_{j=1}^{n} e_{j} .
\end{aligned}
$$

Indeed, let $\sigma \subset\{1, \ldots, n\}$ be such that $|\sigma|>(1-\varepsilon) n$ and the system $\left(x_{j}\right)_{j \in \sigma}$ is $M$-equivalent to an orthogonal basis. By a change of coordinates, the system $\left(x_{j}\right)_{1 \leq j \leq|\sigma|-1}$ must be $M$-equivalent to an orthogonal basis as well. However,

$$
\left\|\sum_{j=1}^{|\sigma|-1} x_{j}\right\|^{2} \leq 2(\varepsilon n+1)
$$

while $\left\|x_{j}\right\| \geq 1 / 2$ for all $j$. Therefore $M$ cannot be bounded independently of $n$ as $\varepsilon \rightarrow 0$. This proves Remark 2 .

3. Almost orthogonal subsequences of frames. In this section we prove an infinite-dimensional version of Theorem 5 .

TheOREM 10. Given an $\varepsilon>0$, every infinite-dimensional frame has a subsequence $(1-\varepsilon)$-equivalent to an orthogonal basis of $l_{2}$.

Given two sets $A$ and $B$ in $H$, we put by definition

$$
\theta(A, B)=\sup _{a \in A} \operatorname{dist}(a, B)=\sup _{a \in A} \inf \{\|a-b\|: b \in B\} .
$$

Lemma 11. Let $\left(x_{j}\right)$ be a frame in an infinite-dimensional $H$. Let $A=$ $\left\{x_{j} /\left\|x_{j}\right\|\right\}$. Then for any finite-dimensional subspace $E \subset H$,

$$
\theta(A, E)=1 \text {. }
$$

Proof. Let $z_{j}=x_{j} /\left\|x_{j}\right\|$ for all $j$. Assume that, on the contrary, there is a $\delta<1$ such that

$$
\operatorname{dist}\left(z_{j}, E\right)<\delta \quad \text { for all } j .
$$

Let $P$ be the orthogonal projection in $H$ onto $E$. Then

$$
\left\|P z_{j}\right\|>\sqrt{1-\delta^{2}} \text { for all } j
$$


so that

$$
\left\|P x_{j}\right\| \geq \sqrt{1-\delta^{2}} \cdot\left\|x_{j}\right\| \quad \text { for all } j .
$$

Since $P$ is finite-dimensional, Lemma 4 yields that the sequence $\left\|P x_{j}\right\|$ is square summable. Then, by (6), $\left\|x_{j}\right\|$ must also be square summable. Thus, from Lemma $4,\left(x_{j}\right)$ is finite-dimensional. This contradiction completes the proof.

Lemma 12. Let $\varepsilon_{j}$ be a sequence of fast decreasing positive numbers $\left(2^{-j-1}\right.$ will do). Let $\left(z_{j}\right)$ be a normalized sequence in $H$ such that

$$
\left\langle z_{i}, z_{j}\right\rangle<\varepsilon_{j} \quad \text { whenever } i<j .
$$

Then $\left(z_{j}\right)$ is equivalent to an orthonormal basis.

The proof is simple.

Proof of Theorem 10. First note that, given an $\varepsilon>0$, every subsequence equivalent to the canonical vector basis of $l_{2}$ is weakly null, therefore has a subsequence which is $(1-\varepsilon)$-equivalent to the canonical vector basis of $l_{2}$. Hence by Corollary 2 we may assume that our given frame $\left(x_{j}\right)$ is tight. Let $z_{j}=x_{j} /\left\|x_{j}\right\|$ for all $j$. We will find a subsequence $\left(z_{j_{k}}\right)$ equivalent to an orthogonal basis by induction. Put $j_{1}=1$. Let $j_{1}, \ldots, j_{k-1}$ be defined and let $E=\operatorname{span}\left(z_{j_{1}}, \ldots, z_{j_{k-1}}\right)$. Choose $j_{k}$ from Lemma 11 so that

$$
\operatorname{dist}\left(z_{j_{k}}, E\right)>1-2^{-2 k} \text {. }
$$

Then it is easy to check that the constructed subsequence $\left(z_{j_{k}}\right)$ satisfies the assumption of Lemma 12. This finishes the proof.

\section{A frame not containing bases with brackets}

Definition 13. A sequence $\left(x_{n}\right)_{n=1}^{\infty}$ in a Banach space $X$ is called a basis with brackets if there are numbers $1<n_{1}<n_{2}<\ldots$ such that every vector $x \in X$ admits a unique representation of the form

$$
x=\lim _{j} \sum_{n=1}^{n_{j}} a_{n} x_{n}, \quad a_{n} \in \mathbb{R} .
$$

Clearly, every basis is a basis with brackets. The difference between bases and bases with brackets is that the latter require the convergence only of some partial sums in the representation.

The following lemma is known $[\mathrm{L}-\mathrm{T}]$.

LEMMA 14. Let $\left(x_{n}\right)_{n=1}^{\infty}$ be a basis with brackets, and numbers $1<n_{1}<$ $n_{2}<\ldots$ be as in Definition 13. Consider the projection $P_{j}$ onto $\left[x_{n}: n \leq n_{j}\right]$ parallel to $\left[x_{n}: n>n_{j}\right]$. Then $\sup _{j}\left\|P_{j}\right\|<\infty$.

Clearly, the converse also holds: if $\sup _{j}\left\|P_{j}\right\|<\infty$ for some sequence $1<n_{1}<n_{2}<\ldots$, then $\left(x_{n}\right)$ is a basis with brackets. 
In this section we prove

THEOREM 15. There exists a frame in $l_{2}$ which does not contain bases with brackets.

Moreover, this frame is tight and has norms bounded from below.

Lemma 16. There is an orthonormal basis $\left(z_{j}\right)$ in $l_{2}^{n}$ such that, given any set $J \subset\{1, \ldots, n\}$ with $|J| \geq n-2$, one has

$$
\begin{aligned}
& \operatorname{dist}\left(e_{1},\left[z_{j}: j \in J, j \geq j_{0}\right]\right) \leq 4 / \sqrt{n} \quad \text { for } 1 \leq j_{0}<n / 2, \\
& \operatorname{dist}\left(e_{n},\left[z_{j}: j \in J, j<j_{0}\right]\right) \leq 4 / \sqrt{n} \quad \text { for } n / 2 \leq j_{0} \leq n .
\end{aligned}
$$

Proof. By rotation, it is enough to find normalized vectors $v_{1}, v_{2}$ in $l_{2}^{n}$ such that $\left\langle v_{1}, v_{2}\right\rangle=0$ and, given a set $J$ as in the hypothesis,

$$
\begin{array}{ll}
\operatorname{dist}\left(v_{1},\left[e_{j}: j \in J, j \geq j_{0}\right]\right) \leq 4 / \sqrt{n} & \text { for } 1 \leq j_{0}<n / 2, \\
\operatorname{dist}\left(v_{2},\left[e_{j}: j \in J, j<j_{0}\right]\right) \leq 4 / \sqrt{n} & \text { for } n / 2 \leq j_{0} \leq n .
\end{array}
$$

Clearly, one may take

$$
\begin{aligned}
& v_{1}=\lceil n / 2\rceil^{-1 / 2} \cdot(\underbrace{1, \ldots, 1}_{\lceil n / 2\rceil}, 0, \ldots, 0), \\
& v_{2}=\lceil n / 2\rceil^{-1 / 2} \cdot(0, \ldots, 0, \underbrace{1, \ldots, 1}_{\lceil n / 2\rceil}) .
\end{aligned}
$$

We will construct our frame $\left(x_{j}\right)$ by defining blocks $\left(x_{j}: j \in J(n)\right)$, where

$$
J(1)=\{1\}, \quad J(2)=\{2,3\}, \quad J(3)=\{4,5,6\}, \quad J(4)=\{7,8,9,10\}, \ldots
$$

The supports of $x_{j}$ 's from block $J(n)$ will lie in an interval $I(n)$, where

$$
I(1)=\{1\}, \quad I(2)=\{1,2\}, \quad I(3)=\{2,3,4\}, \quad I(4)=\{4,5,6,7\}, \ldots
$$

Let $i(n)$ be the first element of $I(n)$.

$$
\begin{array}{cccccccccc}
* & * & * & & & & & 0 & & \\
\\
* & * & * & * & * & & & & & \\
& & * & * & * & & & & & \\
& & * & * & * & * & * & * & * & \\
& & & & & * & * & * & * & \\
& 0 & & & & * & * & * & * & \\
& & & & * & * & * & * & \ldots
\end{array}
$$

The columns of this infinite matrix form the frame elements $x_{j}$, the asterisks marking their supports. Consider the shift operator $T_{n}: l_{2}^{n} \rightarrow l_{2}$ which sends $\left(e_{i}\right)_{i=1}^{n}$ to $\left(e_{i}: i \in I(n)\right)$. Choose an orthonormal basis $\left(z_{j}: j \in\right.$ $J(n))$ in $l_{2}^{n}$ satisfying the conclusion of Lemma 16, and define

$$
x_{j}=T_{n} z_{j} \quad \text { for } j \in J(n) .
$$


LEMma 17. $\left(x_{j}\right)$ is a frame in $l_{2}$.

Proof. Indeed, look at the rows in the picture, that is, the vectors $y_{i}=$ $\left(x_{1}(i), x_{2}(i), \ldots\right)$. Since the vectors $x_{j}, j \in J(n)$, are orthonormal for fixed $n$, the vectors $y_{i}$ are orthogonal. Moreover, their norms are either 2 (if $i=i(n)$ for some $n$ ) or 1 (otherwise). Now we pass again from the rows $y_{i}$ to the columns $x_{j}$. Lemma 3 yields that $\left(x_{j}\right)$ is a frame.

Let $J$ be a set of positive integers such that the sequence $\left(x_{j}\right)_{j \in J}$ is complete in $l_{2}$. We shall prove that it is not a basis with brackets.

LEMma 18. $|J(n) \cap J| \geq n-2$ for every $n$.

Proof. Let $P$ be the orthogonal projection onto those $n-2$ coordinates in $I(n)$ which do not belong to the other blocks $I\left(n_{1}\right)$, i.e. onto $\left[e_{i}: i \in\right.$ $I(n) \backslash\{i(n), i(n+1)\}]$. Thus $P$ sends to zero all $x_{j}$ with $j \notin J(n)$. Hence $\operatorname{Im}(P)=P\left(\left[x_{j}: j \in J(n) \cap J\right]\right)$. Since $\operatorname{Im}(P)$ is an $(n-2)$-dimensional space, the lemma follows.

In what follows we consider large blocks $J(n)$, i.e. with $n \rightarrow \infty$. Given a vector $v$ and a subspace $L$ in $l_{2}$ (both possibly depending on $n$ ), we say that $v$ is close to $L$ if $\operatorname{dist}(x, L) \leq c / \sqrt{n}$. Here $c$ is some absolute constant, whose value may be different in different occurrences.

Lemma 19. (1) $e_{i(n)}$ is close to $\left[x_{j}: j \in J(n-1) \cap J\right]$.

(2) $e_{i(n+1)}$ is close to $\left[x_{j}: j \in J(n+1) \cap J\right]$.

(3) For each $j_{0} \in J(n)$, either $e_{i(n)}$ is close to $\left[x_{j}: j \in J(n) \cap J, j \geq j_{0}\right]$, or $e_{i(n+1)}$ is close to $\left[x_{j}: j \in J(n) \cap J, j<j_{0}\right]$.

Proof. Note that $T_{n}$ sends $e_{1}$ to $e_{i(n)}$ and $e_{n}$ to $e_{i(n+1)}$. Then all three statements of the lemma follow from Lemma 16.

The next (and last) lemma, in tandem with Lemma 14, completes the proof of Theorem 15 .

Lemma 20. For every $j_{0} \in J(n)$ there is a normalized vector $x$ in $l_{2}$ which is close to both subspaces $E=\left[x_{j}: j \in J, j \geq j_{0}\right]$ and $F=\left[x_{j}: j \in J\right.$, $\left.j<j_{0}\right]$.

Proof. We make use of Lemma 19. By (3), we take either $x=e_{i(n)}$ to have $x$ close to $E$, or $x=e_{i(n+1)}$ to have $x$ close to $F$. In the first case $x$ is also close to $F$ by (2), and in the second case $x$ is close to $E$ by (1). The proof is complete.

A part of this work was accomplished when the author was visiting Friedrich-Schiller-Universität Jena. The author is grateful to M. Rudelson and P. Wojtaszczyk for helpful discussions, and to V. Kadets for his constant encouragement. 


\section{References}

[A] A. Aldroubi, Portraits of frames, Proc. Amer. Math. Soc. 123 (1995), 1661-1668.

[B-Tz] J. Bourgain and L. Tzafriri, Invertibility of "large" submatrices with applications to the geometry of Banach spaces and harmonic analysis, Israel J. Math. 57 (1987), 137-224.

[C1] P. G. Casazza, Characterizing Hilbert space frames with the subframe property, Illinois J. Math. 41 (1997), 648-666.

[C2] - Local theory of frames and Schauder bases for Hilbert space, ibid. 43 (1999), 291-306.

[C-C1] P. G. Casazza and O. Christensen, Hilbert space frames containing a Riesz basis and Banach spaces which have no subspace isomorphic to $c_{0}$, J. Math. Anal. Appl. 202 (1996), 940-950.

[C-C2] - - - Frames containing a Riesz basis and preservation of this property under perturbations, SIAM J. Math. Anal. 29 (1998), 266-278.

[D-S] R. J. Duffin and A. C. Schaeffer, A class of nonharmonic Fourier series, Trans. Amer. Math. Soc. 72 (1952), 341-366.

[He] C. Heil, Wavelets and Frames. Signal Processing, Part I, IMA Vol. Math. Appl. 22, Springer, 1990, 147-160.

[Ho] J. R. Holub, Pre-frame operators, Besselian frames, and near-Riesz bases in Hilbert spaces, Proc. Amer. Math. Soc. 122 (1994), 779-785.

$[\mathrm{K}-\mathrm{Tz}]$ B. Kashin and L. Tzafriri, Some remarks on the restrictions of operators to coordinate subspaces, preprint.

[L-T] J. Lindenstrauss and L. Tzafriri, Classical Banach Spaces, Springer, 1977.

[L] A. Lunin, On operator norms of submatrices, Mat. Zametki 45 (1989), 94-100 (in Russian).

[S] K. Seip, On the connection between exponential loss and certain related sequences in $L^{2}(-\pi, \pi)$, J. Funct. Anal. 130 (1995), 131-160.

Faculty of Mathematics and Computer Science

The Weizmann Institute of Science

Rehovot 76100, Israel

E-mail: vershyn@wisdom.weizmann.ac.il

Received March 1, 1999

Revised version December 27, 2000 